Pacific Journal of Mathematics

ENUMERATION OF UP-DOWN PERMUTATIONS BY NUMBER
OF RISES 


\section{ENUMERATION OF UP-DOWN PERMUTATIONS BY NUMBER OF RISES}

\section{Carlitz}

It is well known that $A(n)$, the number of up-down permutations of $\{1,2, \cdots, n\}$ satisfies

$$
\begin{aligned}
& \sum_{n=0}^{\epsilon} A(2 n) \frac{z^{2 n}}{(2 n) !}=\sec z, \\
& \sum_{n=0}^{\infty} A(2 n+1) \frac{z^{2 n+1}}{(2 n+1) !}=\tan z .
\end{aligned}
$$

In the present paper generating functions are obtained for the number of up-down permutations counting the number of rises among the "peaks".

1. If $\left(a_{1}, a_{2}, \cdots, a_{n}\right)$ denotes an arbitrary up-down permutation, then $\left(b_{1}, b_{2}, \cdots, b_{n}\right)$, where

$$
b_{i}=n-a_{i}+1 \quad(i=1,2, \cdots, n),
$$

is a down-up permutation and vice versa.

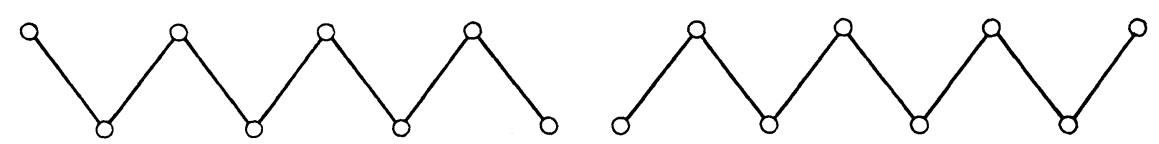

Figure 1

Thus, for $n>1$, there is a one-to-one correspondence between up-down and down-up permutations so that it suffices to consider the former.

Let $A(n, r)$ denote the number of up-down permutations of $Z_{n}=$ $\{1,2, \cdots, n\}$ with $r$ rises on the top line.

Let $C(n, r)$ denote the number of down-up permutations with $r$ rises on the top line.

A rise is a pair of consecutive elements $a, b$ with $a<b$. Also we agree to count a conventional rise on the left. For example

$$
\text { 132546, } 426153
$$

have 3 and 2 rises, respectively.

It will be instructive first to derive the generating functions for $A(2 n+1)$ and $A(2 n)$. We have $A(1)=1$ and

$$
A(2 n+1)=\sum_{k=0}^{n-1}\left(\begin{array}{c}
2 n \\
2 k+1
\end{array}\right) A(2 k+1) A(2 n-2 k-1) \quad(n>0) .
$$

Hence if we put 


$$
F(z)=\sum_{n=0}^{\infty} A(2 n+1) \frac{z^{2 n+1}}{(2 n+1) !},
$$

it follows from (1.1) that

$$
F^{\prime}(z)=1+F^{2}(z)
$$

Since $F(0)=0$, we get $F(z)=\tan z$.

Next

$$
A(2 n)=\sum_{k=0}^{n-1}\left(\begin{array}{l}
2 n-1 \\
2 k+1
\end{array}\right) A(2 k+1) A(2 n-2 k-2),
$$

where $A(0)=1$. Hence if

$$
G(z)=\sum_{n=0}^{\infty} A(2 n) \frac{z^{2 n}}{(2 n) !},
$$

it follows from (1.2) that

$$
G^{\prime}(z)=F(z) G(z) .
$$

Since $G(0)=1$, this gives $G(z)=\sec z$. Thus we have proved that [1], [2, pp. 105-112]

$$
\sum_{n=0}^{\infty} A(n) \frac{z^{n}}{n !}=\sec z+\tan z
$$

2. Turning next to $A(2 n+1, r)$ we take

$$
A(1,0)=1, A(1, r)=0
$$

Corresponding to (1.1) we have the recurrence

(2.1) $A(2 n+1, r)=\sum_{k=0}^{n-1} \sum_{s=0}^{r}\left(\begin{array}{c}2 k+1 \\ 2 n\end{array}\right) A(2 k+1, s) A^{*}(2 n-2 k-1, r-s)$, where

$$
A^{*}(2 n+1, r)=A(2 n+1, r)
$$

but

$$
A^{*}(1,0)=0, A^{*}(1,1)=1 \text {. }
$$

Put

$$
\begin{aligned}
& A_{2 n+1}(x)=\sum_{r} A(2 n+1, r) x^{r}, A_{1}(x)=1 \\
& A_{2 n+1}^{*}(x)=A_{2 n+1}(x) \quad(n>0), A_{1}^{*}(x)=x .
\end{aligned}
$$

Then (2.1) gives 


$$
A_{2 n+1}(x)=\sum_{k=0}^{n-1}\left(\begin{array}{c}
2 k+1 \\
2 n
\end{array}\right) A_{2 k+1}(x) A_{2 n-2 k-1}^{*}(x) \quad(n>0) .
$$

Hence if

$$
A(z)=A(x, z)=\sum_{n=0}^{\infty} A_{2 n+1}(x) \frac{z^{2 n+1}}{(2 n+1) !},
$$

it follows from (2.2) that

$$
\begin{aligned}
A^{\prime}(z) & =\sum_{n=0}^{\infty} A_{2 n+1}(x) \frac{z^{2 n}}{(2 n) !} \\
& =1+\sum_{k=0}^{\infty} A_{2 k+1}(x) \frac{z^{2 k+1}}{(2 k+1) !} \sum_{n=1}^{\infty} A_{2 n-1}^{*}(x) \frac{z^{2 n-1}}{(2 n-1) !},
\end{aligned}
$$

so that

$$
\begin{aligned}
A^{\prime}(z) & =1+A(z)(A(z)-(1-x) z) \\
& =1-(1-x) z A(z)+A^{2}(z) .
\end{aligned}
$$

If we put

$$
A(z)=\frac{1}{U} \frac{d U}{d z}, \frac{d A}{d z}=\frac{1}{U^{2}}\left(\frac{d U}{d z}\right)^{2}-\frac{1}{U} \frac{d^{2} U}{d z^{2}},
$$

(2.4) becomes

$$
\frac{d^{2} U}{d z^{2}}+(1-x) z \frac{d U}{d z}+U=0
$$

It is clear that $U$ is an even function of $z$. We accordingly put

$$
U=\sum_{n=0}^{\infty}(-1)^{n} a_{n}(x) \frac{z^{2 n}}{(2 n) !} \quad\left(a_{0}(x)=1\right) .
$$

Substituting in (2.5) we get

$$
-a_{n+1}(x)+2 n(1-x) a_{n}(x)+a_{n}(x)=0,
$$

so that

$$
a_{n+1}(x)=(1+2 n(1-x)) a_{n}(x) .
$$

It follows at once from (2.6) that

$$
a_{n}(x)=\prod_{k=0}^{n-1}(1+2 k(1-x)) .
$$

Hence

$$
U=\sum_{n=0}^{\infty}(-1)^{n} \prod_{k=0}^{n=1}(1+2 k(1-x)) \cdot \frac{z^{2 n}}{(2 n) !},
$$


and

$$
A(z)=\frac{\sum_{n=0}^{\infty}(-1)^{n} \prod_{k=0}^{n}(1+2 k(1-x)) \cdot \frac{z^{2 n+1}}{(2 n+1) !}}{\sum_{n=0}^{\infty}(-1)^{n} \prod_{k=0}^{n-1}(1+2 k(1-x)) \cdot \frac{z^{2 n}}{(2 n) !}}
$$

The first few coefficients are given by

$$
A_{1}(x)=1, A_{3}(x)=2 x, A_{5}(x)=8 x+8 x^{2}, A_{7}(x)=48 x+176 x^{2}+48 x^{3} .
$$

It follows by induction from

$$
A_{2 n+1}(x)=\sum_{k=1}^{n-2}\left(\begin{array}{c}
2 n+1 \\
2 k
\end{array}\right) A_{2 k+1}(x) A_{2 n-2 k-1}(x)+2 n(1+x) A_{2 n-1}(x) \quad(n>1)
$$

that

$$
x^{n+1} A_{2 n+1}\left(\frac{1}{x}\right)=A_{2 n+1}(x)
$$

This implies

$$
A(2 n+1, r)=A(2 n+1, n-r+1) \quad(1 \leqq r \leqq n) .
$$

Also, using the fuller notation $A(x, z)$, we have

$$
x^{1 / 2} A\left(\frac{1}{x}, x^{1 / 2} z\right)=(x-1) z+A(z, x) .
$$

3. Now we consider the case $A(2 n, r)$. We take

$$
A(0,0)=1, A(0, r)=0
$$

Corresponding to (1.2) we have the recurrence

$$
\begin{array}{r}
A(2 n+2, r)=\sum_{k=0}^{n} \sum_{s}\left(\begin{array}{l}
2 n+1 \\
2 k+1
\end{array}\right) A(2 k+1, s) A^{*}(2 n-2 k, r-s) \\
(n \geqq 0),
\end{array}
$$

where

$$
A^{*}(2 n, r)=A(2 n, r) \quad(n>0),
$$

but

$$
A^{*}(0,0)=0, A^{*}(0,1)=1 \text {. }
$$

Now put 


$$
\begin{aligned}
& A_{2 n}(x)=\sum_{r} A(2 n, r) x^{r}, A_{0}(x)=1, \\
& A_{2 n}^{*}(x)=A_{2 n}(x)(n>0), A_{0}^{*}(x)=x .
\end{aligned}
$$

Then (3.1) gives

$$
A_{2 n+2}(x)=\sum_{k=0}^{n}\left(\begin{array}{c}
2 n+1 \\
2 k+1
\end{array}\right) A_{2 k+1}(x) A_{2 n-2 k}^{*}(x) \quad(n \geqq 0) .
$$

Hence if

$$
B(z)=B(x, z)=\sum_{n=0}^{\infty} A_{2 n}(x) \frac{z^{2 n}}{(2 n) !},
$$

we have

$$
B^{\prime}(z)=A(z)(B(z)-1+x) .
$$

Replacing $A(z)$ by $U^{\prime} / U$, we get

$$
U B^{\prime}+U^{\prime} B=(1-x) U^{\prime}
$$

Since $B(0)=1, U(0)=1$, it follows from (3.4) that

$$
U B=x+(1-x) U \text {. }
$$

Therefore

$$
B(z)=1-x+\frac{x}{U}
$$

The first few coefficients are

$$
A_{0}(x)=A_{2}(x)=x, A_{4}(x)=3 x+2 x^{2}, A_{6}(x)=15 x+38 x^{2}+8 x^{3} .
$$

4. We turn now to $C(2 n, r)$. We take

$$
C(0,0)=1, C(0, r)=0 \quad(r>0) .
$$

We have the recurrence

$$
C(2 n+2, r)=\sum_{k=0}^{n} \sum_{s}\left(\begin{array}{c}
2 n+1 \\
2 k
\end{array}\right) C(2 k, s) A^{*}(2 n-2 k+1, r-s),
$$

where $A^{*}(2 k+1, s)$ has the same meaning as in $\S 2$.

Thus, if

$$
C_{2 n}(x)=\sum_{r} C(2 n, r) x^{r}
$$

we get

$$
C_{2 n+2}(x)=\sum_{k=0}^{n}\left(\begin{array}{c}
2 n+1 \\
2 k
\end{array}\right) C_{2 k}(x) A_{2 n-2 k+1}^{*}(x)
$$


Put

$$
C(z)=C(x, z)=\sum_{n=0}^{\infty} C_{2 n}(x) \frac{z^{2 n}}{(2 n) !}
$$

Then it follows from (4.2) that

$$
C^{\prime}(z)=C(z)(A(z)-(1-x) z),
$$

so that

$$
\frac{C^{\prime}(z)}{C(z)}=\frac{U^{\prime}}{U}-(1-x) z
$$

Since $C(0)=1$, this yields

$$
C(z)=\frac{1}{U} e^{-1 / 2(1-x) z^{2}}
$$

The first few coefficients are

$C_{0}(x)=1, C_{2}(x)=x, C_{4}(x)=2 x+3 x^{2}, C_{6}(x)=8 x+38 x^{2}+15 x^{3}$.

We shall now show that $U=U(x, z)$ satisfies the functional equation

$$
U\left(\frac{1}{x}, x^{1 / 2} z\right) e^{-1 / 2(1-x) z^{2}}=U(x, z),
$$

or

$$
\sum_{0}^{\infty}(-1)^{n} a_{n}\left(\frac{1}{x}\right) \frac{x^{n} z^{2 n}}{(2 n) !} \sum_{0}^{\infty}(-1)^{k} \frac{(1-x)^{k} z^{2 k}}{2^{k} \cdot k !}=\sum_{0}^{\infty}(-1)^{n} a_{n}(x) \frac{z^{2 n}}{(2 n) !} .
$$

This is equivalent to

$$
\sum_{k=0}^{n} \frac{(2 n) !}{(2 k) !(n-k) !}\left(\frac{1-x}{2}\right)^{n-k} a_{k}\left(\frac{1}{x}\right)=a_{n}(x) .
$$

The left hand side of (4.7) is equal to

$$
\begin{aligned}
& \frac{(2 n) !}{n !}\left(\frac{1-x}{2}\right)^{n} \sum_{k=0}^{n}(-1)^{k} \frac{(-n)_{k}}{k !\left(\frac{1}{2}\right)_{k}}\left(\frac{x}{2(1-x)}\right)^{k} \prod_{j=0}^{k-1}\left(1+2 j\left(1-\frac{1}{x}\right)\right) \\
& =\frac{(2 n) !}{n !}\left(\frac{1-x}{2}\right)^{n} \sum_{k=0}^{n} \frac{(-n)_{k}}{k !\left(\frac{1}{2}\right)_{k}}\left(\frac{x}{2(k-1)}\right)_{k} \\
& =\frac{(2 n) !}{n !}\left(\frac{1-x}{2}\right)^{n} \frac{\left(\frac{1}{2(1-x)}\right)_{n}}{\left(\frac{1}{2}\right)_{n}}=2^{n}(1-x)^{n}\left(\frac{1}{2(1-x)}\right)_{n}=a_{n}(x),
\end{aligned}
$$


by Vandermonde's theorem.

It evidently follows from (3.5), (4.5), and (4.6) that

$$
C_{2 n}(x)=x^{n+1} A_{2 n}\left(\frac{1}{x}\right)
$$

and therefore

$$
C(2 n, r)=A(2 n, n-r+1) \quad(1 \leqq r \leqq n) .
$$

5. Finally we consider $C(2 n+1, r)$. We now take

$$
C(1,1)=1, C(1, r)=0 \quad(r \neq 1) .
$$

We have the recurrence

$$
C(2 n+1, r)=\sum_{k=0}^{n} \sum_{s}\left(\begin{array}{l}
2 n \\
2 k
\end{array}\right) C(2 k) A^{*}(2 n-2 k, r-s) .
$$

Thus, if

$$
C_{2 n+1}(x)=\sum_{r} C(2 n+1, r) x^{r}
$$

it follows that

$$
C_{2 n+1}(x)=\sum_{k=0}^{n}\left(\begin{array}{l}
2 n \\
2 k
\end{array}\right) C_{2 k}(x) A_{2 n-2 k}^{*}(x)
$$

Put

$$
D(z)=D(x, z)=\sum_{n=0}^{\infty} C_{2 n+1}(x) \frac{z^{2 n+1}}{(2 n+1) !} .
$$

Then, by (5.2),

$$
D^{\prime}(z)=C(z)(B(z)-1+x) .
$$

It follows that

$$
\begin{aligned}
D^{\prime}(z) & =\frac{x}{U^{2}(x, z)} e^{-1 / 2(1-x) z^{2}} \\
& =\frac{x}{U(x, z) U\left(x^{-1}, x^{1 / 2} z\right)} \\
& =x C(x, z) C\left(x^{-1}, x^{12} z\right) .
\end{aligned}
$$

Therefore

$$
C_{2 n+1}(x)=\sum_{k=0}^{n}\left(\begin{array}{l}
2 n \\
2 k
\end{array}\right) x^{n-k+1} C_{2 k}(x) C_{2 n-2 k}\left(x^{-1}\right) .
$$

It follows from (5.4) that 


$$
x^{n+2} C_{2 n+1}\left(x^{-1}\right)=C_{2 n+1}(x),
$$

so that

$$
C(2 n+1, r)=C(2 n+1, n-r+2) \quad(1 \leqq r \leqq n+1) .
$$

The first few values of $C_{2 n+1}+(x)$ are given by

$$
\begin{aligned}
C_{1}(x) & =x, C_{3}(x)=x+x^{2}, C_{5}(x)=3 x+10 x^{2}+3 x^{3}, C_{7}(x) \\
& =15 x+121 x^{2}+121 x^{3}+15 x^{4}
\end{aligned}
$$

Note that $C_{2 n+1}(x)$ is of degree $n+1$.

6. A number of special values can be obtained. It follows first from

$$
A_{2 n+1}(x)=\sum_{k=1}^{n-2}\left(\begin{array}{c}
2 n \\
2 k+1
\end{array}\right) A_{2 k+1}(x)+2 n(1+x) A_{2 n-1}(x) \quad(n>1)
$$

and

$$
x \mid A_{2 k+1}(x) \quad(k>0)
$$

that

$$
A_{2 n+1}^{\prime}(0)=2 n A_{2 n-1}^{\prime}(0)
$$

This yields

$$
A(2 n+1,1)=2 n A(2 n-1,1)=2^{n} n ! .
$$

Next, it follows from

$$
A_{2 n+2}(x)=(2 n+1) A_{2 n}(x)+\sum_{k=1}^{n-1}\left(\begin{array}{l}
2 n+1 \\
2 k+1
\end{array}\right) A_{2 k+1}(x) A_{2 n-2 k}(x)+x A_{2 n+1}(x)
$$

and

$$
x \mid A_{2 k}(x) \quad(k>0)
$$

that

$$
A_{2 n+2}^{\prime}(0)=(2 n+1) A_{2 n}^{\prime}(0) \text {. }
$$

This gives

$$
A(2 n, 1)=(2 n-1)(2 n-3) \cdots 3.1 .
$$

It follows from

$$
\begin{aligned}
C_{2 n+2}(x)= & A_{2 n+1}(x)+\sum_{k=1}^{n-1}\left(\begin{array}{c}
2 n+1 \\
2 k
\end{array}\right) C_{2 k}(x) A_{2 n-2 k+1}(x) \\
& +(2 n+1) x C_{2 n}(x)
\end{aligned}
$$


and

$$
x \mid C_{2 k}(x) \quad(k>0)
$$

that

$$
C_{2 n+2}^{\prime}(x)=A_{2 n+1}^{\prime}(0) \quad(n>0)
$$

Hence

$$
C(2 n+2,1)=2^{n} n !
$$

Finally, from

$$
C_{2 n+1}(x)=A_{2 n}(x)+\sum_{k=1}^{n-1}\left(\begin{array}{l}
2 n \\
2 k
\end{array}\right) C_{2 k}(x) A_{2 n-2 k}(x)+x C_{2 n}(x)
$$

and

$$
x \mid C_{2 k+1}(x) \quad(k \geqq 0),
$$

we get

$$
C_{2 n+1}^{\prime}(0)=A_{2 n}^{\prime}(0)
$$

so that

$$
C(2 n+1,1)=(2 n-1)(2 n-3) \cdots 3.1 .
$$

In view of (4.9),

$$
C(2 n, r)=A(2 n, n-r+1) \quad(1 \leqq r \leqq n),
$$

we have also

$$
\begin{gathered}
A(2 n+2, n+1)=2^{n} n ! \\
C(2 n, n)=(2 n-1)(2 n-3) \cdots 3.1 .
\end{gathered}
$$

7. We remark that the differential equation

$$
U^{\prime \prime}+(1-x) z U^{\prime}+U=0
$$

has the second solution $W=U V$, where

$$
V^{\prime}=e^{-1 / 2(1-x) z^{2}} U^{-2} .
$$

Thus, by (5.3), we have

$$
D^{\prime}(z)=x V^{\prime}(z)
$$

so that

$$
D(z)=x V(z)
$$


Since $W$ is an odd function of $z$ we may put

$$
W(z)==\sum_{0}^{\infty}(-1)^{n} b_{n}(x) \frac{z^{2 n+1}}{(2 n+1) !} .
$$

It follows from the differential equation that

$$
b_{n+1}(x)=(1+(2 n+1)(1-x)) b_{n}(x),
$$

so that

$$
b_{n}(x)=\prod_{k=0}^{n-1}(1+(2 k+1)(1-x)) .
$$

Finally we have

$$
D(z)=x \frac{\sum_{n=0}^{\infty}(-1)^{n} \prod_{k=0}^{n-1}(1+(2 k+1)(1-x)) \cdot \frac{z^{2 n+1}}{(2 n+1) !}}{\sum_{n=0}^{\infty}(-1)^{n} \sum_{k=0}^{n-1}(1+2 k(1-x)) \cdot \frac{z^{2 n}}{(2 n) !}} .
$$

We may, if we prefer, express both $U$ and $V$ as hypergeometric functions of the type ${ }_{1} F_{1}$.

\section{REFERENCES}

1. R. C. Entringer, A combinatorial interpretation of the Euler and Bernoulli numbers, Nieuw Archief voor Wiskunde (3), 14 (1966), 241-246.

2. E. Netto, Lehrbuch der Combinatorik, Teubner, Leipzig and Berlin, 1927.

Received November 17, 1971. Supported in part by NSF grant GP-17031.

DUKE UNIVERSITY 


\section{PACIFIC JOURNAL OF MATHEMATICS}

EDITORS

\author{
H. SAMELSON \\ Stanford University \\ Stanford, California 94305 \\ C. R. HOBBY \\ University of Washington \\ Seattle, Washington 98105
}

\author{
J. DUGUNDJI \\ Department of Mathematics \\ University of Southern California \\ Los Angeles, California 90007 \\ RICHARD ARENS \\ University of California \\ Los Angeles, California 90024
}

\section{ASSOCIATE EDITORS}

E. F. BECKENBACH

B. H. NeUManN

F. WOLF

K. YosHIDA

\section{SUPPORTING INSTITUTIONS}

UNIVERSITY OF BRITISH COLUMBIA

CALIFORNIA INSTITUTE OF TECHNOLOGY

UNIVERSITY OF CALIFORNIA

MONTANA STATE UNIVERSITY

UNIVERSITY OF NEVADA

NEW MEXICO STATE UNIVERSITY

OREGON STATE UNIVERSITY

UNIVERSITY OF OREGON

OSAKA UNIVERSITY
UNIVERSITY OF SOUTHERN CALIFORNIA

STANFORD UNIVERSITY

UNIVERSITY OF TOKYO

UNIVERSITY OF UTAH

WASHINGTON STATE UNIVERSITY UNIVERSITY OF WASHINGTON

AMERICAN MATHEMATICAL SOCIETY NAVAL WEAPONS CENTER 


\section{Pacific Journal of Mathematics}

\section{Vol. 45, No. $1 \quad$ September, 1973}

William George Bade, Complementation problems for the Baire classes .......... 1

Ian Douglas Brown, Representation of finitely generated nilpotent groups ........ 13

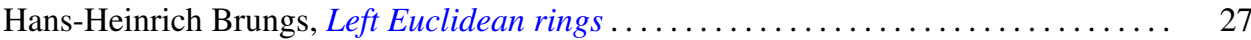

Victor P. Camillo and John Cozzens, A theorem on Noetherian hereditary rings ..... 35

James Cecil Cantrell, Codimension one embeddings of manifolds with locally flat

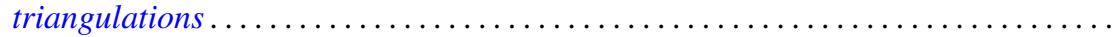

L. Carlitz, Enumeration of up-down permutations by number of rises . . . . . . . . . .

Thomas Ashland Chapman, Surgery and handle straightening in Hilbert cube

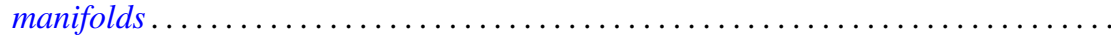

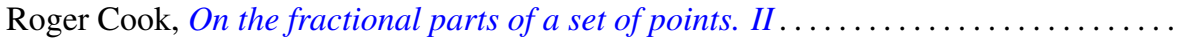

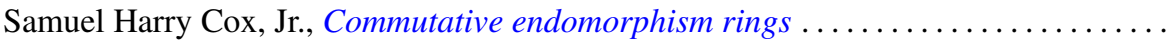

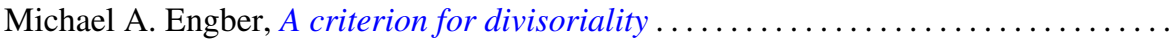

Carl Clifton Faith, When are proper cyclics injective . . . . . . . . . . . . . . 97

David Finkel, Local control and factorization of the focal subgroup . . . . . . . . . 113

Theodore William Gamelin and John Brady Garnett, Bounded approximation by

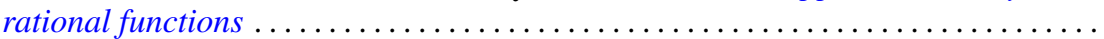

Kazimierz Goebel, On the minimal displacement of points under Lipschitzian

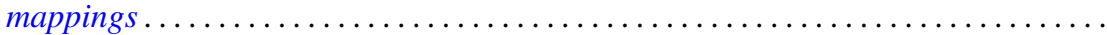

Frederick Paul Greenleaf and Martin Allen Moskowitz, Cyclic vectors for representations associated with positive definite measures: nonseparable

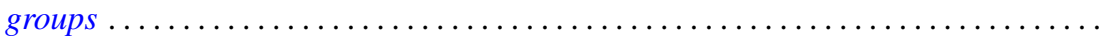

Thomas Guy Hallam and Nelson Onuchic, Asymptotic relations between perturbed linear systems of ordinary differential equations .

David Kent Harrison and Hoyt D. Warner, Infinite primes of fields and completions. .

James Michael Hornell, Divisorial complete intersections . ......

Jan W. Jaworowski, Equivariant extensions of maps ..............

John Jobe, Dendrites, dimension, and the inverse arc function .. .

Gerald William Johnson and David Lee Skoug, Feynman integrals of non-factorable

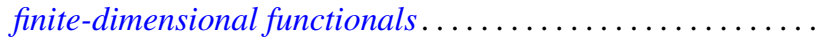

Dong S. Kim, A boundary for the algebras of bounded holomorphic functions ...... 269

Abel Klein, Renormalized products of the generalized free field and its derivatives ... 275

Joseph Michael Lambert, Simultaneous approximation and interpolation in $L_{1}$ and

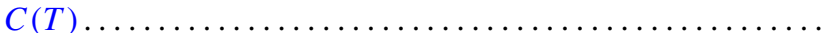

Kelly Denis McKennon, Multipliers of type $(p, p)$ and multipliers of the group

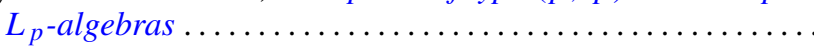

William Charles Nemitz and Thomas Paul Whaley, Varieties of implicative

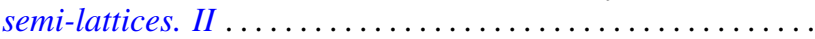

Donald Steven Passman, Some isolated subsets of infinite solvable

Norma Mary Piacun and Li Pi Su, Wallman compactifications on E-completely

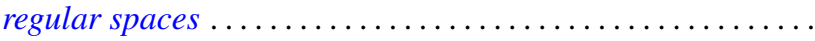

Jack Ray Porter and Charles I. Votaw, $S(\alpha)$ spaces and regular Hausdorff extensions....

Gary Sampson, Two-sided $L_{p}$ estimates of convolution transforms .

Ralph Edwin Showalter, Equations with operators forming a rig
Raymond Earl Smithson, Fixed points in partially ordered sets .

Victor Snaith and John James Ucci, Three remarks on symmetric products and

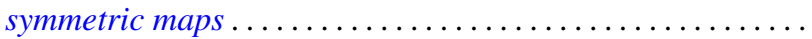

